J. Gynäkol. Endokrinol. AT 2021 · 31:34-38 https://doi.org/10.1007/s41974-021-00176-w Angenommen: 4. Februar 2021

@ Der/die Autor(en) 2021

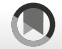

\section{Christoph Brezinka}

Department Frauenheilkunde, Universitätsklinik für Gynäkologie und Geburtshilfe, Medizinische Universität Innsbruck, Innsbruck, Österreich

\title{
Risikoberechnung von Ovarialzysten nach IOTA mit der ADNEX-App
}

Eine Zyste an einem der Eierstöcke, die bei einer Ultraschalluntersuchung festgestellt wurde, führt vielfach zu einem operativen Eingriff, vor allem bei der postmenopausalen Patientin. Das rasche Wachstum und das hohe Malignitätspotenzial von Ovarialtumoren scheinen ein zügiges Handeln nahezulegen, an dessen Ende meist die allgemeine Erleichterung steht, dass die Zyste doch nicht bösartig war. Die in der Rückschau oft nicht wirklich notwendigen Operationen zahlreicher „Ultraschallzysten“ führte zu einer Geringschätzung der Methode

\begin{tabular}{|c|c|}
\hline A Back Patient & ata \\
\hline $\begin{array}{l}\text { Age of the patient } \\
\text { at examination }\end{array}$ & 84 \\
\hline $\begin{array}{l}\text { Oncology center } \\
\text { (referral center for } \\
\text { gyn-oncol)? }\end{array}$ & Yes \\
\hline $\begin{array}{l}\text { Maximal diameter } \\
\text { of the lesion }\end{array}$ & 96 \\
\hline $\begin{array}{l}\text { Maximal diameter } \\
\text { of the largest solid } \\
\text { part }\end{array}$ & 5 \\
\hline $\begin{array}{l}\text { More than } 10 \\
\text { locules? }\end{array}$ & No \\
\hline $\begin{array}{l}\text { Number of } \\
\text { papillations } \\
\text { (papillary } \\
\text { projections) }\end{array}$ & None \\
\hline $\begin{array}{l}\text { Acoustic shadows } \\
\text { present? }\end{array}$ & No \\
\hline $\begin{array}{l}\text { Ascites (fluid } \\
\text { outside pelvis) }\end{array}$ & No \\
\hline
\end{tabular}

Abb. 1 A Eingabe der Daten des Falles von - Abb. 3 in die Smartphone-App von IOTAADNEX der gynäkologischen Vaginalsonographie. Mit Einführung der IOTAKriterien erlebt der gynäkologische Ultraschall eine Qualitätsverbesserung, ähnlich wie 20 Jahre zuvor die Einführung der Nackenfaltenmessung dem Pränatalultraschall zu allgemeiner Akzeptanz verhalf. Mit dem ADNEX-Modell, einem an Tausenden Patientinnenverläufen validierten Mehrklassenrisikomodell, ist es nun möglich, unmittelbar im Anschluss an die Ultraschalluntersuchung mit der Patientin ihr individuelles Risiko zu besprechen.

Neu aufgetretene Zysten der Ovarien, die im Zuge einer gynäkologischen Routineuntersuchung im Ultraschall gesehen werden, müssen einer gezielten und strukturierten Diagnostik zugeführt werden. Allerdings führt auch ein als diagnostisch deklarierter operativer Eingriff fast unweigerlich $\mathrm{zu}$ einem Verlust an
Ovargewebe, etwa bei einem als Zyste angesprochenen Corpus luteum. Die weite Verbreitung des Ultraschalls in der Gynäkologie und der Fertigkeitserwerb im Rahmen chaotischer Autodidaktik führte ab Ende der 1980er-Jahre zum „Syndrom des sichtbaren Ovars" und vermeidbaren Verlusten von Ovargewebe bei Frauen im fertilen Lebensalter [1]. Andererseits kann ein abwartendes Vorgehen bei einem im Ultraschall früh aufgefallenen Ovarialkarzinom diesem und seiner malignen Tumorbiologie einen therapeutisch nicht mehr einzuholenden Vorsprung verschaffen.

Seit 20 Jahren arbeitet die IOTAGruppe vom belgischen Leuven aus an der Etablierung plausibler, robuster und reproduzierbarer Kriterien für die Beurteilung von Adnexbefunden im Vaginalultraschall [2]. In Weiterentwicklung der bekannten "IOTA simple rules" wurde das computergestützte Berechnungsmodell Assessment of Different Neoplasias

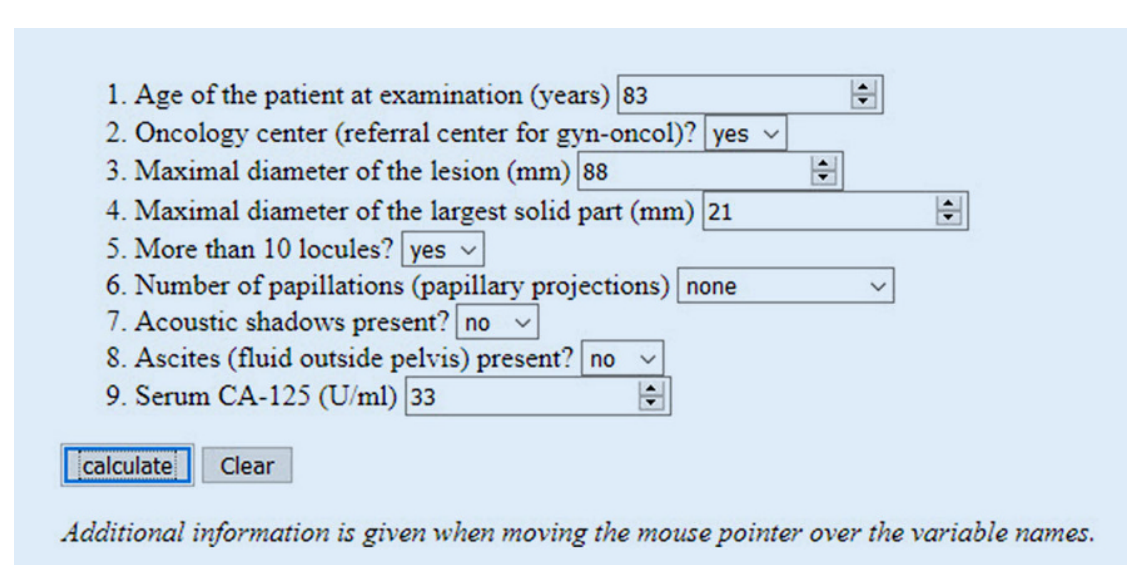

Abb. $2 \Delta$ Eingabe der Daten von $\triangle$ Abb. 9 in die Gratis-Web-Applikation des ADNEX-Modells auf der IOTA-Website (www.iotagroup.org) 


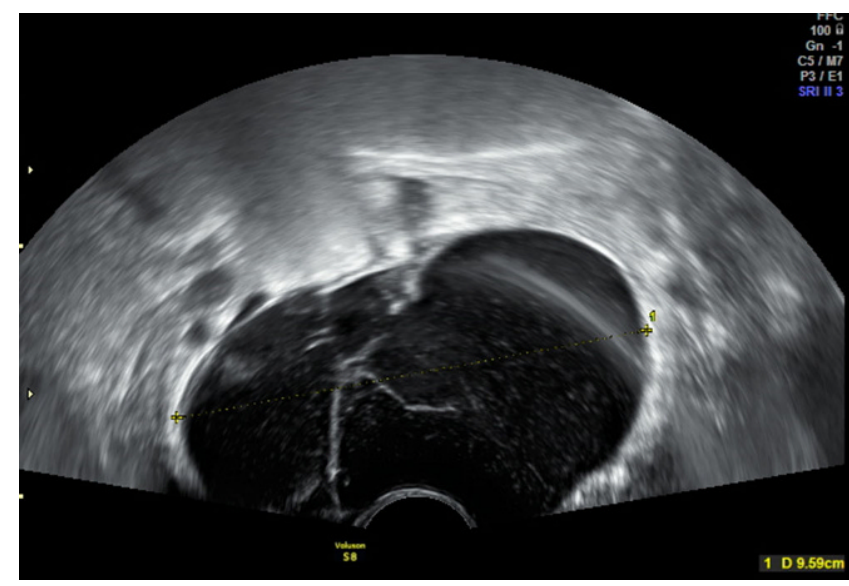

Abb. 3 ॥ Bei einer 84-jährigen Patientin betrug der Maximaldurchmesser der Läsion $96 \mathrm{~mm}$. Der größte solide Anteil war $5 \mathrm{~mm}$, weniger als 10 Loculi, keine papillären Projektionen, keine Verschattungen und kein Aszites. Das ADNEX-Risiko eines malignen Tumors betrug 15,2\%. Eine laparoskopische Operation ergab ein seröses Zystadenom

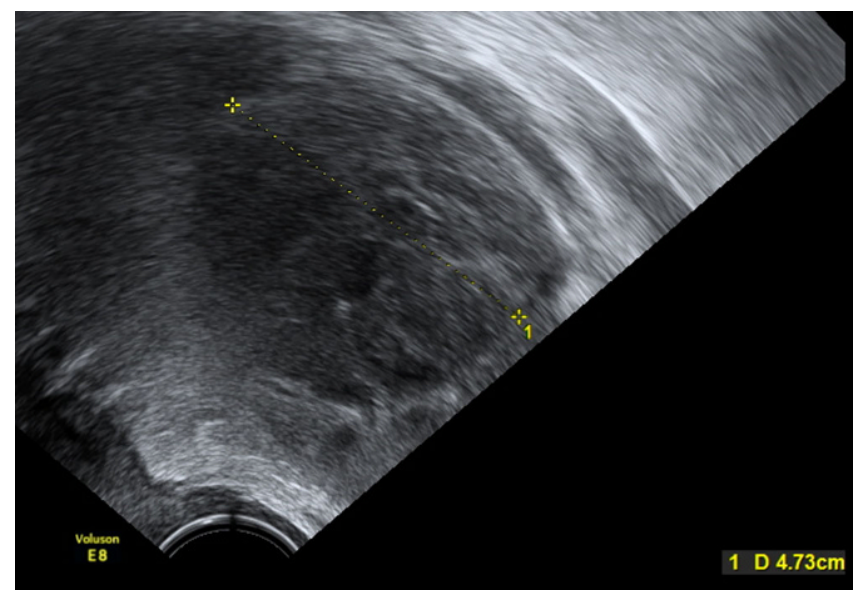

\section{Abb. $5<$ Ein} $49 \mathrm{~mm}$ großer solider Tumor im Adnexbereich ergab in ADNEX ein 77,7\%iges Risiko der Malignität. Der Tumor stellte sich als die Metastase eines Melanoms heraus

in the $\operatorname{adnEXa}$ (ADNEX) entwickelt. Dieses ist bereits fix auf der neueren Generation vieler Ultraschallgeräte installiert. Daneben kann man es kostenlos auf der Website der IOTA-Gruppe in Löwen in Belgien nutzen (www.iotagroup. org) und es sich gegen einen geringen Betrag als App aufs Smartphone laden. ADNEX ist ein Mehrklassenvorhersagemodell, welches hilft, im Anschluss an die Ultraschalluntersuchung zwischen gutartigen und bösartigen Tumoren zu differenzieren. Weiters bietet ADNEX eine Subklassifikation in Borderline-Tumoren, primäre Karzinome in den Stadien I-IV und sekundär metastatische (Krukenberg-)Tumoren der Ovarien [3].

\section{Anwendung des ADNEX-Modells}

Das Modell beschränkt sich auf die Ovarien, nur Abweichungen von der Normalität, die in der IOTA-Diktion „Läsionen“ sind, werden überhaupt eingegeben. Das heißt, eine als Corpus luteum erkannte Struktur, also ein physiologischer Befund, sollte nicht zu einer Läsion ernannt werden. Nicht dem Ovar zuordenbare Strukturen wie gestielte Myome, TarlovZysten, Darm- und Blasentumoren sollten auch nicht mit ADNEX analysiert werden.

Sechs Prädiktoren des ADNEX-Modells basieren auf Ultraschall, auf Beurteilungen und Messungen. Im Rahmen der Ultraschalluntersuchung müssen die Kriterien und Messgrößen, aus denen diese Prädiktoren entstehen, schrittweise „ab-

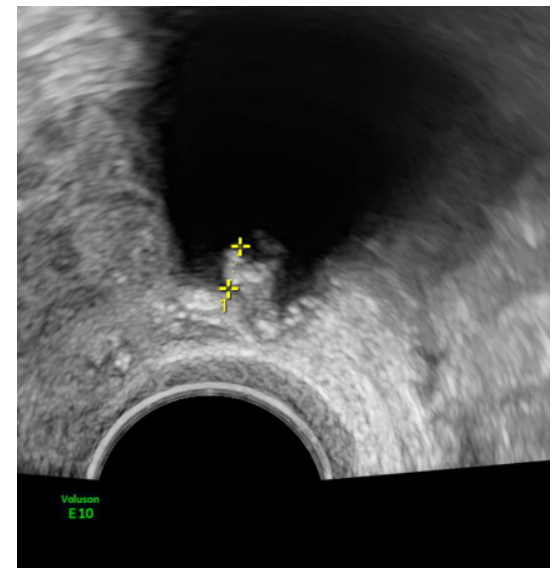

Abb. $6 \Delta$ Beispiel für papilläre Projektion. Die gemessene Strecke beträgt 4,4 mm. Es ist wichtig, nur die papilläre Projektion zu messen und nicht auch den Zystenrand

gearbeitet" und am Ende der Untersuchung in das Modell eingegeben werden (- Abb. 1 und 2). Die Art der Messung ist durch die bekannten "simple rules“ von IOTA vorgegeben [4].

Zuerst wird der Gesamtdurchmesser der Läsion in drei Dimensionen gemessen und der größte Durchmesser eingegeben (• Abb. 1). Der nächste Schritt besteht darin, den soliden Anteil innerhalb der Läsion zu messen (• Abb. 4). Dieser kann - bei soliden Tumoren - identisch mit dem Gesamtdurchmesser sein, oder 


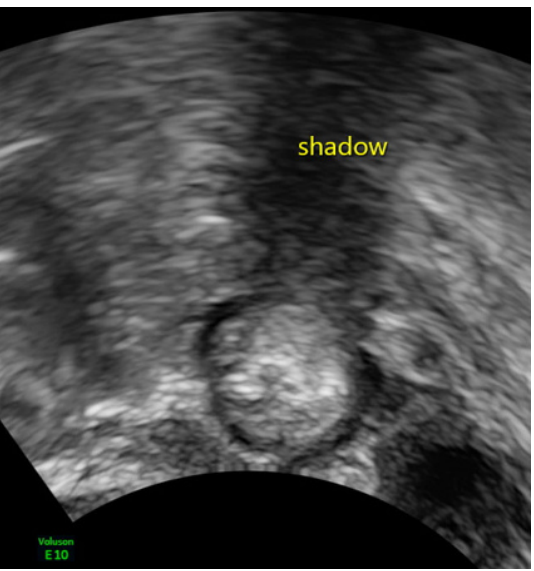

Abb. $7 \Delta$ Beispiel für "shadowing" distal eines $18 \mathrm{~mm}$ großen Dermoids. Diese Verschattungen ergeben in ADNEX "mildernde Umstände" und senken das kalkulierte Risiko

\begin{tabular}{|c|c|c|}
\hline \& Bacl & Results & Home $\mathbf{0}$ \\
\hline 】 ${ }_{\mathrm{C}}^{\mathrm{R}}$ & $\begin{array}{l}\text { Risk of Metastatic } \\
\text { Cancer to the } \\
\text { Adnexa }\end{array}$ & $2.3 \%$ \\
\hline I $\mathrm{R}$ & $\begin{array}{l}\text { Risk of stage II-IV } \\
\text { Ovarian cancer }\end{array}$ & $4.5 \%$ \\
\hline $\begin{array}{l}\mathrm{R} \\
\mathrm{C}\end{array}$ & $\begin{array}{l}\text { Risk of stage I } \\
\text { Ovarian cancer }\end{array}$ & $3.7 \%$ \\
\hline $\begin{array}{l}R \\
T\end{array}$ & $\begin{array}{l}\text { Risk of Borderline } \\
\text { Tumor }\end{array}$ & $4.7 \%$ \\
\hline - $\begin{array}{l}\mathrm{R} \\
\mathrm{N}\end{array}$ & $\begin{array}{l}\text { Risk of } \\
\text { Malignancy }\end{array}$ & $15.2 \%$ \\
\hline$\rightarrow \begin{array}{l}C \\
B\end{array}$ & $\begin{array}{l}\text { Chance of } \\
\text { Benign Tumor }\end{array}$ & $84.8 \%$ \\
\hline & Check the Charts & \\
\hline
\end{tabular}

Abb. $9 \Delta$ Ergebnis der Risikokalkulation in der Smartphone-App von IOTA-ADNEX auf Basis der Daten von $-A b b .3$ und 1

auch ein Nullwert, wenn die Läsion nur aus einer oder mehreren Zysten besteht (• Abb. 5).

Wichtig ist auch die Anzahl der Zysten: In dem etwas archaischen Englisch von IOTA ist eine einzelne Zyste ein „Locule“. Mehrere Zysten sind „Locules“, bei Anwendung von ADNEX wird gefragt, ob bei der Untersuchung mehroder weniger als 10 Zysten-„Locules“ gezählt wurden.

Die „papillary projections" sind mehr als $3 \mathrm{~mm}$ große Vorwölbungen vom Zys-

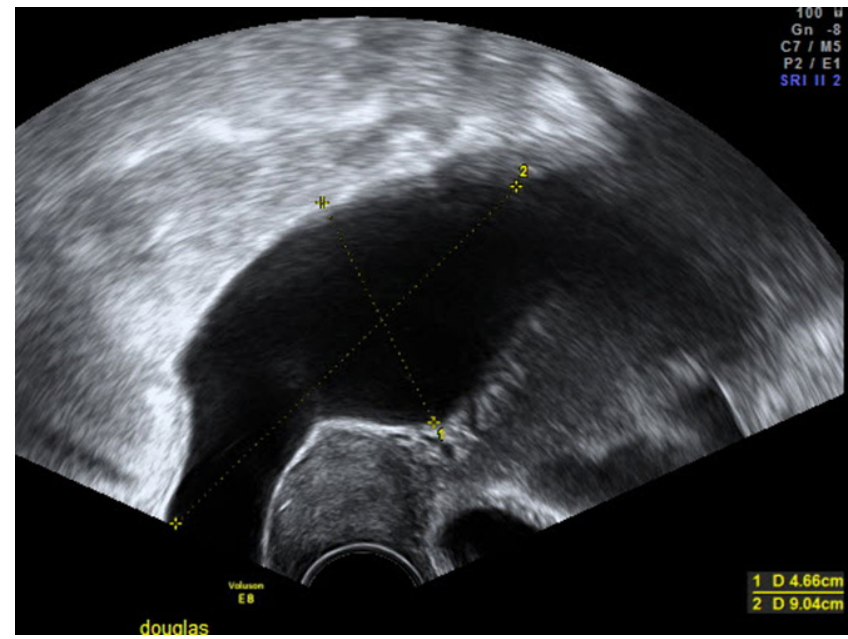

Abb. $8 \triangleleft$ Beispiel für Aszites: Dieser Befund muss in der ADNEX-Fragenliste mit "yes" angegeben werden und führt zu einer Erhöhung des kalkulierten Risikos

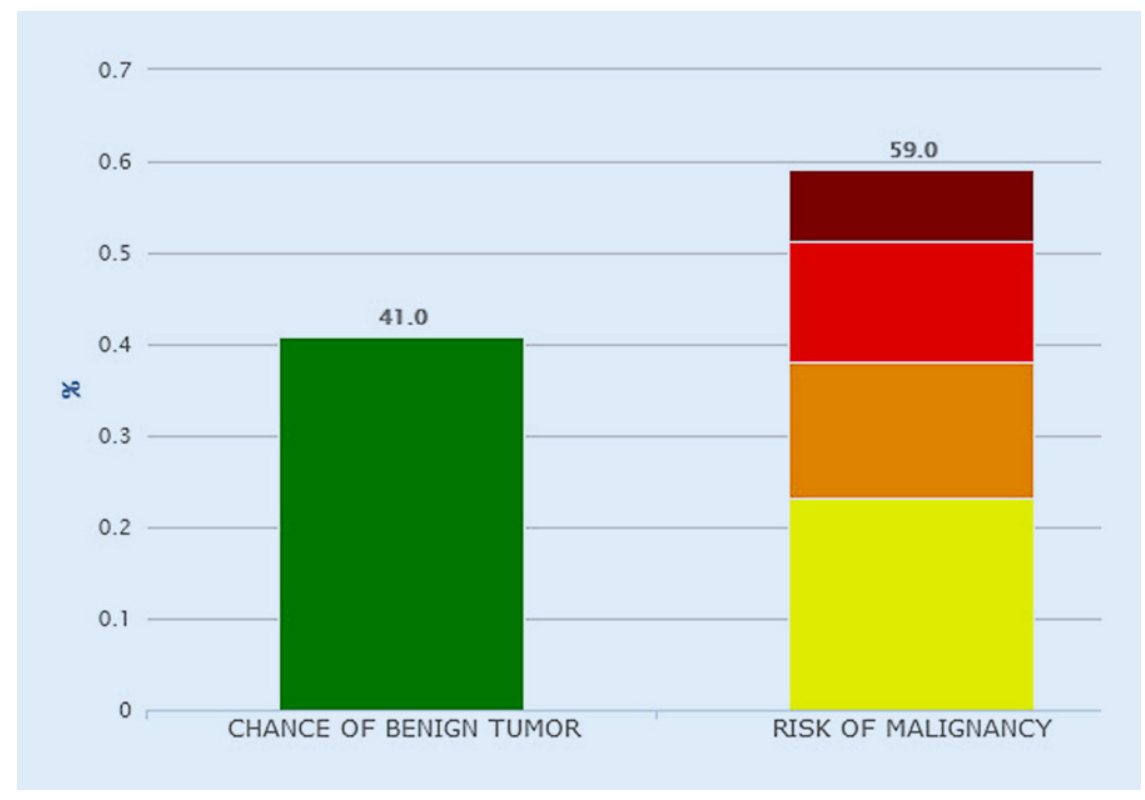

Abb. 10 ॥ Grafische Darstellung der Auswertung der Daten von $-A b b .9$ in der Gratis-Web-Applikation des ADNEX-Modells auf der IOTA-Website (www.iotagroup.org)

tenrand ins Zystenlumen (• Abb. 6). Die Anzahl dieser Vorwölbungen im Untersuchungsgebiet muss gezählt werden. Sind diese kleiner als $3 \mathrm{~mm}$, so gelten sie als Unregelmäßigkeit der Zystenwand.

Als Nächstes ist auf akustische Schatten $\mathrm{zu}$ achten, die die Läsion allenfalls verursachen kann (• Abb. 7). Schließlich muss das Vorhandensein von Aszites registriert werden $(\bullet \mathbf{A b b} .8) . \mathrm{Zu}$ den Nichtultraschallwerten zählt zunächst das Alter der Patientin, dann muss eingegeben werden, ob die Untersuchung an einem gynäkologisch-onkologischen Schwerpunktzentrum stattfindet (• Abb. 1).
Schließlich sollte noch der CA-125Wert eingegeben werden. Dieser ist nicht obligat, vor der Berechnung des Risikos warnt das System jedoch, dass die Genauigkeit mit CA 125 höher wäre. Dieser Wert kann auch an einem folgenden Tag eingegeben und der Befund entsprechend modifiziert und präzisiert werden (- Abb. 9 und 10). Von allen im Zuge von IOTA untersuchten Biomarkern hat sich CA 125 als der verlässlichste herausgestellt [5].

Die Entscheidung, was mit den Daten der Risikoeinschätzung gemacht wird, hängt von Alter, Komorbiditäten, Wünschen und Perspektiven der Patientin ab, weiters von den Ressourcen, die in ei- 


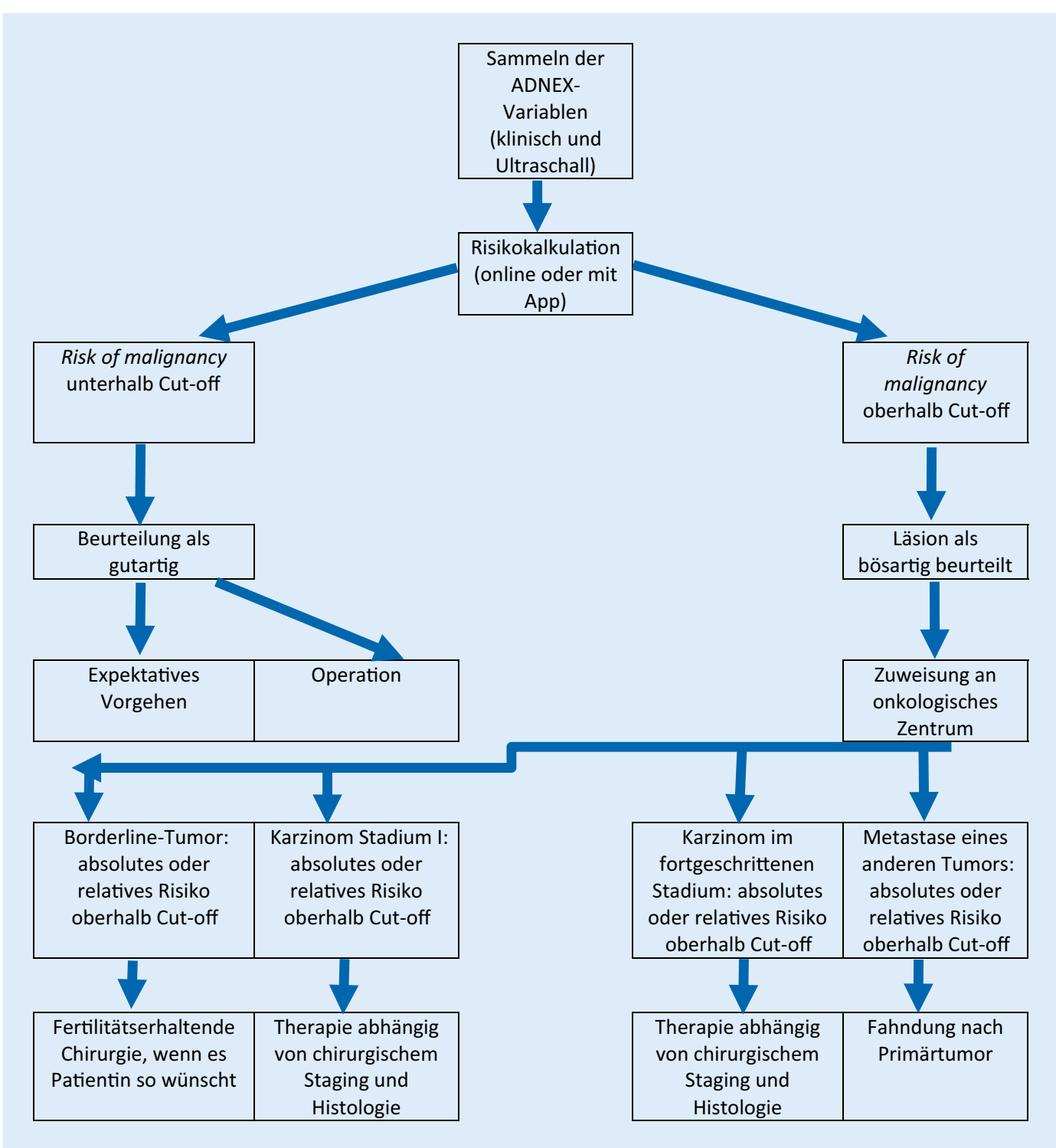

Abb. $11<$ Einsatz des ADNEX-Modells in mehreren diagnostischen Schritten [6]

\section{Weiterführende Informationen \\ Mehr zu IOTA, Online-Kursen, Prüfungen und Zertifizierungen unter: www.iotagroup.org}

nem Gesundheitssystem zur Verfügung stehen (Schema • Abb. 11) [6].

Die IOTA-Gruppe hat für den gynäkologischen Schall erreicht, was die Nackenfaltendiagnostik in den 1990erJahren für den Schwangerenultraschall und die Pränataldiagnostik erreicht hat. Es war im Pränatalultraschall nun nicht mehr allein die Gemengelage aus Erfahrung, Gefühl und Tagesverfassung des einzelnen Untersuchers ausschlaggebend, es gab messbare und erlernbare Ultraschallkriterien, die in ein validier- tes Risikoberechnungsmodell einflossen. In der Pränataldiagnostik führten die reproduzierbare Messung spezifischer Strukturen und die Hinzunahme biochemischer Serummarker zu einer Unterstützung bei der Entscheidung, ob eine invasive Untersuchung vorgenommen werden sollte oder nicht.

Verbesserung der Ultraschallgeräte, Training und Verbesserung der Fertigkeiten der Untersucher sowie Verbesserung der Trennschärfe der Software durch Studien mit Zehntausenden Patientinnen haben das Ersttrimesterscreening zu einer standardisierten Untersuchung in den meisten europäischen Schwangerschaften gemacht.
Die IOTA-Kriterien sind an vielen Zentren bereits ein fester Bestandteil der präoperativen Untersuchungen und ein wichtiger Faktor für die Indikationsstellung für Eingriffe an den Adnexen. Mit ihrer nun erfolgten Übernahme im traditionell ultraschallskeptischen Nordamerika ist $\mathrm{zu}$ erwarten, dass sich weltweit ein verlässliches Tumorscreeningprogramm entwickelt [7]. Das ADNEXModell wird sicher in den nächsten Jahren durch genauere und noch besser validierte Risikoberechnungen abgelöst werden. Derzeit ist es ein einfach einzusetzendes Werkzeug, das die Entscheidung und Beratung bei Adnexprozessen wesentlich erleichtert. 


\section{Korrespondenzadresse}

Ao. Univ.-Prof. Dr. Christoph Brezinka

Department Frauenheilkunde, Universitäts-

klinik für Gynäkologie und Geburtshilfe,

Medizinische Universität Innsbruck

Anichstraße 35, 6020 Innsbruck, Österreich

christoph.brezinka@i-med.ac.at

Funding. Open access funding provided by University of Innsbruck and Medical University of Innsbruck.

\section{Einhaltung ethischer Richtlinien}

Interessenkonflikt. C. Brezinka gibt an, dass kein

Interessenkonflikt besteht.

Für diesen Beitrag wurden vom Autor keine Studien an Menschen oder Tieren durchgeführt. Für die aufgeführten Studien gelten die jeweils dort angegebenen ethischen Richtlinien.

Open Access. Dieser Artikel wird unter der Creative Commons Namensnennung 4.0 International Lizenz veröffentlicht, welche die Nutzung, Vervielfältigung, Bearbeitung, Verbreitung und Wiedergabe in jeglichem Medium und Format erlaubt, sofern Sie den/die ursprünglichen Autor(en) und die Quelle ordnungsgemäß nennen, einen Link zur Creative Commons Lizenz beifügen und angeben, ob Änderungen vorgenommen wurden.
Die in diesem Artikel enthaltenen Bilder und sonstiges Drittmaterial unterliegen ebenfalls der genannten Creative Commons Lizenz, sofern sich aus der Abbildungslegende nichts anderes ergibt. Sofern das betreffende Material nicht unter der genannten Creative Commons Lizenz steht und die betreffende Handlung nicht nach gesetzlichen Vorschriften erlaubt ist, ist für die oben aufgeführten Weiterverwendungen des Materials die Einwilligung des jeweiligen Rechteinhabers einzuholen.

Weitere Details zur Lizenz entnehmen Sie bitte der Lizenzinformation auf http://creativecommons.org/ licenses/by/4.0/deed.de.

\section{Literatur}

1. Zalud I, Busse R, Funduk-Kurjak B (2013) Asymptomatic simple ovarian cyst in postmenopausal women: syndrome of the 'visible ovary'. Donald Sch JUltrasound Obstet Gynecol 7:182-186

2. Timmerman $D$, Testa $A C$, Bourne $T$, Ferrazzi $E$, Ameye L, Konstantinovic ML, Van CB, Collins WP Vergote I, Van HS, Valentin L (2005) Logistic regression model to distinguish between the benign and malignant adnexal mass before surgery: a multicenter study by the International Ovarian Tumor Analysis Group. J Clin Oncol 23(34):8794-8801

3. Van Calster B, Valentin L, Froyman W, Landolfo C, Ceusters J, Testa AC, Wynants L, Sladkevicius $P$ Van Holsbeke C, Domali E, Fruscio R, Epstein E, Franchi D, Kudla MJ, Chiappa V, Alcazar JL, Leone FPG, Buonomo F, Coccia ME, Guerriero S, Deo N,
Jokubkiene L, Savelli L, Fischerova D, Czekierdowski A, Kaijser J, Coosemans A, Scambia G, Vergote I, Bourne T, Timmerman D (2020) Validation of models to diagnose ovarian cancer in patients managed surgically or conservatively: multicentre cohort study. BMJ 370:m2614

4. Timmerman $D$, Testa $A C$, Bourne $T$, Ameye $L$, Jurkovic D, Van HC, Paladini D, Van CB, Vergote I, Van HS, Valentin L (2008) Simple ultrasoundbased rules for the diagnosis of ovarian cancer. Ultrasound Obstet Gynecol 31(6):681-690

5. Medeiros LR, Rosa DD, da Rosa MI, Bozzetti MC (2009) Accuracy of CA 125 in the diagnosis of ovarian tumors: a quantitative systematic review. Eur J Obstet Gynecol Reprod Biol 142(2):99-105

6. Van Calster B, Van Hoorde K, Froyman W, Kaijser J, Wynants L, Landolfo C, Anthoulakis C, Vergote I, Bourne T, Timmerman D (2015) Practical guidance for applying the ADNEX model from the IOTA group to discriminate between different subtypes of adnexal tumors. Facts Views Vis Obgyn 7(1):32-41

7. Andreotti RF, Timmerman D, Strachowski LM, Froyman W, Benacerraf BR, Bennett GL, Bourne T, Brown DL, Coleman BG, Frates MC, Goldstein SR, Hamper UM, Horrow MM, Hernanz-Schulman M Reinhold C, Rose SL, Whitcomb BP, Wolfman WL Glanc P (2020) O-RADS US risk stratification and management system: a consensus guideline from the ACR Ovarian-Adnexal Reporting and Data System Committee. Radiology 294(1):168-185

Hinweis des Verlags. Der Verlag bleibt in Hinblick auf geografische Zuordnungen und Gebietsbezeichnungen in veröffentlichten Karten und Institutsadressen neutral.

Hier steht eine Anzeige.

\section{Springer}

